\title{
FECUNDIDADE DE Goniopsis cruentata (LATREILLE, 1803) (CRUSTACEA, Brachyura, Grapsidae) no manguezal do rio Paripe - Pernambuco - BRASIL.
}

\author{
Noely Fabiana Oliveira de MOURA ${ }^{1}$ \\ PETRÔNIO AlVEs COELHO ${ }^{1}$
}

1 - UFPE. Dept ${ }^{0}$ de Oceanografia. Av. Arquitetura, s/n Cidade Universitária - Recife, Pernambuco / Brasil. CEP 50790-540. E. mail: nfom@hotlink.com.br

\begin{abstract}
RESUMO
O caranguejo grapsideo Goniopsis cruentata (Latreille, 1803) é comum e abundante nas zonas entremarés de muitos estuários no estado de Pernambuco, nordeste do Brasil, sendo importante fonte de renda para população ribeirinha. Este estudo objetiva caracterizar a fecundidade de G. cruentata no litoral norte de Pernambuco, Brasil. As amostras foram coletadas mensalmente de agosto 1999 a julho 2000 com um artefato de pesca artesanal, com esforço de pesca de 1 hora. No laboratório foram medidas largura da carapaça e abdome (LC; LA). Foram examinadas 45 fêmeas ovígeras com ovos no estágio inicial de desenvolvimento. Utilizou-se o método de subamostragem para contagem dos ovos. Fêmeas ovígeras ocorreram durante todo período de amostragem. Fecundidade variou de 47.000 ovos para fêmeas ovígeras com $30.5 \mathrm{~mm}$ LC a 171.000 ovos para fêmeas com $45.4 \mathrm{~mm}$ LC. A média de número de ovos $(87,000 \pm 25)$ foi alto quando comparado a outros grapsideos. Existe uma correlação entre fecundidade e largura da carapaça $\left(y=0,035 . x^{2,14} ; r=0,79 ; p<0,05\right)$ e largura do abdome $\left(y=0,17 . x^{1,95} ; r=0,74 ; p<0,05\right)$, como para maioria dos Brachyura.
\end{abstract}

Palavra chaves: Fecundidade, Grapsidae, Goniopsis cruentata

\begin{abstract}
Fecundity of Goniopsis cruentata (Latreille, 1803) (Crustacea, Brachyura, Grapsidae) in the rio Paripe mangrove - Pernambuco - Brasil

The grapsid crab Goniopsis cruentata (Latreille, 1803) is common and abundant in the intertidal zone of many estuaries in Pernambuco State, northeastern Brazil, where this species supports local fishery. This study aims to characterize the fecundity of G. cruentata from northern coast of Pernambuco, Brazil. Monthly sampling was performed during full moon, from August 1999 to July 2000 with a artizanal fishing gear, with 1 hour effort per sampling period. In laboratory carapace and abdomen width (CW, AW) of the individuals was measured. Only data from 45 females carrying early stage eggs were considered. The number of eggs was counted after subsampling. The presence of ovigerous females of $G$. cruentata during the whole sampling period. Fecundity estimates ranged from 47,000 eggs per ovigerous females with $30.5 \mathrm{~mm}$ CW to 171,000 eggs per females with $45.4 \mathrm{~mm}$ CW. The mean number of eggs $(87,000+25)$ was high when comparing to other grapsid crabs. Fecundity is correlated with carapace $\left(y=0,035 . x^{2,14}\right.$; $r=0,79 ; \mathrm{p}<0,05)$ and abdomen $\left(\left(y=0,17 \cdot \mathrm{x}^{1,95} ; \mathrm{r}=0,74 ; \mathrm{p}<0,05\right)\right.$ width, so the number of eggs increased with the females' size.
\end{abstract}

Key words: Fecundity, Grapsidae, Goniopsis cruentata

Tropical Oceanography, Recife: v. 31, n.2, p. 127-133, 2003 


\section{INTRODUÇÃO}

Goniopsis cruentata distribui-se desde Flórida (EUA) até as proximidades do estado de Santa Catarina (Brasil) (Sternberg, 1994; Melo, 1996). Habitam os manguezais do supralitoral ao mediolitoral, tanto em substratos consolidados como nos inconsolidados (Coelho, 1965; Coelho \& Ramos-Porto, 1995).

Segundo Du Preez \& McLachlan (1984), existe uma ampla variação do número de ovos produzido pelos crustáceos, que está associada ao processo evolutivo da espécie e adaptação ao meio. Um dos meios de avaliar o potencial reprodutivo da espécie e/ou o tamanho do estoque populacional é através da fecundidade (Fonteles-Filho, 1989) que, adaptado para os anomuros, braquiúros e outros representantes dos Decapoda é explicado como o número de ovos exteriorizados por fêmea, por desova, que se encontram aderidos aos pleópodos (Somerton \& Meyers, 1983; Valenti et al., 1989; Negreiros-Fransozo et al., 1992). Assim a fecundidade também oferece subsídios para o entendimento da estratégia reprodutiva, já que é um dos componentes de ajuste, o qual é dependente do desempenho reprodutivo durante o ciclo de vida (Sastry, 1983).

Assim, o objetivo deste trabalho está em determinar a fecundidade de Goniopsis cruentata na população do manguezal do rio Paripe, norte da costa do estado de Pernambuco, Brasil.

\section{MAterial e Métodos}

As coletas foram realizadas mensalmente, ao longo do estuário do rio Paripe - Itamaracá - Pernambuco no período de agosto/1999 a julho/2000 durante o período de lua cheia. G. cruentata foi coletado com o auxílio de uma vara a qual prendia-se uma linha de nylon cuja extremidade estava presa uma isca, a exemplo pedaços de peixe, pele da galinha, caranguejos menores, Aratus pisonii e Uca sp.. Como padrão foi utilizado um pescador por 1 hora em cada amostragem. Após a coleta as fêmeas ovígeras foram acondicionadas em sacos plásticos individualmente para tentar evitar a perda da massa de ovos aderida aos pleópodos.

De um total de 664 indivíduos capturados, apenas 45 fêmeas ovígeras com massas de ovos no estágio inicial foram utilizadas para estimar a fecundidade. Os pleópodos de cada fêmea ovígera foram cortados no ponto de inserção com o abdome e transferidos a uma placa de Petri. Para a dissociação dos ovos, foi utilizada uma solução de hipoclorito de sódio a $100 \%$ durante dez segundos e então lavadas em água corrente. Os ovos foram colocados em um becker com 100ml de álcool a 70\%, e com auxílio de uma pipeta graduada retiradas cinco subamostras de $1 \mathrm{ml}$, com reposição. A fecundidade estimada de cada espécime foi obtida multiplicando a média do número de ovos contados pelo total do volume da amostra $(100 \mathrm{ml})$ e dividindo pelo volume da subamostra (Choy, 1988; Hines, 1988; Ruffino et al, 1994).

Além disso, foram determinadas relações entre a fecundidade e a largura da carapaça (LC) e largura do abdome (LA). Para a construção de diagramas de dispersão dos pontos empíricos, foi dotada a equação do tipo potência $\mathrm{Y}=\mathrm{a} \mathrm{X}^{\mathrm{b}}$, onde $\mathrm{Y}=$ número de ovos e $\mathrm{X}=\mathrm{LC}$, ou LA.

\section{RESUltados}

A média da largura da carapaça das 45 fêmeas ovígeras utilizadas para análise de fecundidade foi de 37,6 $\pm 4 \mathrm{~mm}$ e a largura do abdome foi de $23,5 \pm 2,5 \mathrm{~mm}$.

A fecundidade variou de 47.000 ovos para fêmea ovígera com 30,5mm de LC a 171.000 ovos em fêmea de 45,4mm de LC. O valor da fecundidade média foi de $87.000 \pm 25$ ovos.

Na tabela I estão representados os valores da fecundidade média das fêmeas ovígeras de Goniopsis cruentata por classes de tamanho. Através de análise de correlação de Pearson $(\alpha=$ 
$0,05)$ foi possível verificar que existe uma correlação direta com elevada significância estatística (r $=0,94 ; \mathrm{p}<0,05$ ), o que permite predizer qual será a fecundidade individual de uma fêmea de $G$. cruentata com um determinado tamanho de largura da carapaça.

O número de ovos por fêmeas mostrou a tendência de aumentar com a largura da carapaça e pode ser descrito pela relação: $y=0,035 \cdot x^{2,14}$. O coeficiente de correlação foi de 0,79 $(\mathrm{p}<0,05)$ (Figura 1).

Para a relação número de ovos por fêmeas e largura do abdome, também foi verificado um aumento equivalente, com uma equação descrita: $y=0,17 \cdot x^{1,95}$. O coeficiente de correlação foi de $0,74(\mathrm{p}<0,05)$ (Figura 2).

\section{DISCUSSÃO E CONCLUSÃo}

A fecundidade registrada por Goniopsis cruentata no estuário do rio Paripe foi superior ao registrado por Cobo (1999) para a mesma espécie no litoral norte de São Paulo. E quando comparada aos demais grapsídeos apresentou uma nítida superioridade, conforme pode ser observado na tabela II. Infere-se que esta diferença pode estar atribuída a características genéticas das populações, como também a condições fisiológicas das fêmeas, sazonalidade, latitude e condições ambientais como temperatura, salinidade e disponibilidade de alimento (Coelho et al., 1982; Diaz \& Conde, 1989; Valenti et al. 1989). Esta análise comparativa torna-se difícil, considerando inicialmente, os fatores ambientais aos quais estão sujeitas as populações distintas, e as variações no tamanho das espécies.

A alta fecundidade observada em $G$. cruentata certamente corresponde à produção de ovos de pequeno tamanho. Esta fecundidade elevada geralmente está associada a alta mortalidade das larvas e um desenvolvimento larval longo (Rabalais, 1991 apud Sampedro et al., 1997; Reyes et al., 2001). Dessa forma, a alta fecundidade pode indicar que poucas larvas atingem a maturidade (Branco \& Avilar, 1992).

Acredita-se que a presente espécie possa apresentar um ciclo reprodutivo intenso e de extrema eficiência na área estudada, mesmo prevendo a alta mortalidade larval, haja visto o seu sucesso colonizador na região, que pode ser comprovada pela abundância de indivíduos, de cordo com Moura et. al (2000)

Com relação ao tamanho do animal, foi observado para G. cruentata que o número de ovos produzido por fêmea aumenta nitidamente com seu tamanho, como também foi observado por Cobo(1999). Segundo Hines (1982), o tamanho do corpo da fêmea é o principal elemento determinante do potencial reprodutivo nos Brachyura. Assim, as fêmeas de maior tamanho produzem número maior de ovos por desova. Diaz \& Conde (1989) também observaram o aumento do número de ovos com a largura da carapaça para o grapsídeo $A$. pisonii, assim como Baird (1978), analisando o também grapsídeo Sesarma catenata. Na relação entre o número de ovos e a largura do abdome em G. cruentata, o coeficiente de correlação também foi significativo. No que concerne a presença dessas relações, Hines (1982) menciona como sendo o resultado da disponibilidade de espaço reservado para alocação dos recursos utilizados na produção dos ovos, o que nos caranguejos se restringe ao volume interno da cavidade cefalotorácica.

Variação do número de ovos também foi observada dentro de uma mesma classe de tamanho, o que também foi encontrado por Cobo (1999). Este fato pode estar relacionado às perdas acidentais dos ovos como, por exemplo, quando a fêmea está se locomovendo e com isso promove o atrito dos ovos contra o solo (Hines, op cit.; Ruffino et al., 1994); a doenças ou o parasitismo por parte de nemertinos, turbelários e anfípodos cuja infestação pode provocar a perda de até 30\% na massa de ovos (Wickham, 1979; Kuris \& Wickham, 1987; Elner \& Beninger, 1992); ou ainda a existência de desovas múltiplas, ou seja, a exteriorização de mais de uma massa de ovos a partir de uma única copulação, que diminui o número de ovos nas desovas subseqüentes (Hartnoll, 1969; Branco \& Avilar, 1992; Mantelatto \& Fransozo, 1997; Cobo, op cit.). No presente

Tropical Oceanography, Recife: v. 31, n.2, p. 127-133, 2003 
estudo não foi possível averiguar as causas de variação de fecundidade dentro das classes de tamanho. O que se pode concluir sobre a fecundidade em G. cruentata para a área estudada é que esta espécie exibe fecundidade elevada quando comparada a outras espécies da família Grapsidae e que existe relação significativa entre fecundidade e tamanho do animal, como para a maioria dos Brachyura.

\section{Agradecimentos}

À todos que fazem parte do Laboratório de Carcinologia do Departamento de Oceanografia da Universidade Federal de Pernambuco. Ao Conselho Nacional de Desenvolvimento Científico e Tecnológico (CNPq) e a Fundação de Amparo à Ciência e Tecnologia (FACEPE).

\section{REFERÊNCIAS BIBLIOGRÁGICAS}

BAIRD, D. Fecundity of Sesarma catenata Ortmann (Grapsidae, Crustacea) occurring in the saltmarshes of the Swatkops Estuary, Port Elizabeth. South African of the Journal of Science. v.34, p.31-32, 1978.

BRANCO, J.O.; AVILAR, M.G. Fecundidade em Callinectes danaei Smith (Decapoda, Portunidae) da Lagoa da Conceição, Florianópolis, Santa Catarina, Brasil. Revista Brasileira de Zoologia v. 9, n.3-4, p.167-173, 1992

CHOY, S.C..Reproductive biology of Liocarcinus puber and Liocarcinus holsatus (Decapoda, Brachyura, Portunidae) from the Gower Peninsula, South Wales. Marine Ecology v. 9, n. 3, p. $227-241,1988$.

COBO, V. J. Estratégia reprodutiva do caranguejo de manguezal Goniopsis cruentata (LATREILLE, 1803) (Brachyura, Grapsidae) na região de Ubatuba, Litoral Norte do Estado de São Paulo. São Paulo: 1999, 90f. Tese (Doutorado em Zoologia) - Instituto de Biociências da Universidade Estadual Paulista, São Paulo.

COELHO, P. A. Os crustáceos decápodos de alguns manguezais pernambucanos. Trabalhos do Instituto Oceanográfico da Universidade Federal de Pernambuco, v.7, p. 71- 90, 1965.

COELHO, P. A; RAMOS-PORTO, M. Distribuição ecológica dos crustáceos decápodas marinhos do nordeste do Brasil. Trabalhos Oceanográficos da Universidade Federal de Pernambuco v. 23, p.113 - 127, 1995.

COELHO, P.A; RAMOS-PORTO, M.; SOARES, C. M. A. Biologia e cultivo de camarões de água doce. Série Aquicultura, n. 1, Recife: Editora Universitária da UFPE. 1982, 53p.

DIAZ, H.; CONDE, J.E. Population dynamics and life history of the mangrove crab Aratus pisonii (Brachyura, Grapsidae) in a marine environment. Bulletin of Marine Science. v. 45, n. 1, p.148 163, 1989.

DU PREEZ, H. H; MCLACHLAN,A. Biology of three-spot swinming crab Ovalipes punctatus (De Hann). III. Reproduction, fecundity and egg development. Crustaceana v. 47, n.3, p. 285 297. 1984.

Tropical Oceanography, Recife: v. 31, n.2, p. 127-133, 2003 
ELNER,R.W.; BENINGER, P.G. The reproductive biology of snow crab, Chionoecetes opilio: a synthesis of recent contributions. American Zoologist. v.32, p. 524 - 533, 1992.

FONTELES-FILHO, A.A. Recursos pesqueiros, biologia e dinâmica populacional. Imprensa Oficial do Ceará. Fortaleza: 1989, 296p.

HARTNOLL, R.G. Mating in the Brachyura. Crustaceana v. 16, p.162 - 181, 1969.

HINES, A. H. Allometric constraints and variables of reproductive effort in brachyuran crabs. Marine Biology v. 69, p. 309 - 320, 1982.

KURIS, A.M; WICKHAM, D. E. Effect of nemertean egg predators on crustaceans. Bulletin of Marine Science v. 41, p. $151-$ 164, 1987.

MANTELATTO, F. L. M; FRANSOZO, A. Fecundity of the crab Callinectes ornatus Ordway, 1863 (Decapoda, Brachyura, Portunidae) from the Ubatuba region, São Paulo, Brazil.

Crustaceana v. 70, n. 2, p.214 - 226, 1997.

MELO, G. A. S. Manual de identificação dos Brachyura (caranguejos e siris) do litoral brasileiro. Plêiade / FAPESP. São Paulo: 1996, 604p.

MOURA, N. F. O.; COELHO FILHO, P. A.; COELHO, P. A. Population Structure of Goniopsis cruentata (latreille, 1803) in the Paripe Estuary, Brazil. Nauplius, v.8, n. 1, p. 73 - 78, 2000.

NEGREIROS-FRANSOZO, M.L.; FRANSOZO, A; MANTELATTO, F. L. M.; NAKAGAKI, J. M.; SPILBORGHS, M.C.F. Fecundity of Paguristes tortugae Schmitt, 1933 (Crustacea, Decapoda, Anomura) in Ubatuba (SP), Brazil. Revista Brasileira de Biologia v. 52, n.4, p.547553, 1992.

REYES, I.M.H.; FERNÁNDEZ, J.L.P.; BOLAÑOS, J.A.C.; HERNÁNDEZ, J.E. Aspectos reproductivos de Mithrax forceps (A. Milne Edwards, 1875) (Crustacea: Decapoda: Majidae) Ciencias Marinas v. 27, n. 1, p. 21 - 34, 2001.

RUFFINO, M.L.; TELLES, M.D.; D’INCAO, F. Reproductive aspects of Chasmagnathus granulata Dana, 1851 (decapoda, Grapsidae) in the Patos lagoon estuary - Brazil. Nauplius v. 2, p. $43-52,1994$.

SAMPEDRO, M.P.; FERNÁNDEZ, L.; FREIRE, J.; GONZÁLEZ-GURRIARÁN, E. Fecundity and reproductive outup of Pisidia longicornis (Decapoda, Anomura) in the Ría de Arousa (Galicia, NW Spain). Crustaceana v.70, n.1, p. 95 - 109. 1997.

SASTRY, N.A..Ecological aspects of reproduction. In: WATERMAN, TH. (ed) The Biology of Crustacea. VII. Environmental adaptations. Academic Press Inc, 1983, p. 179-270.

SOMERTON, D.A.; MEYERS, W.S. Fecundity diferences between primiparous and multiparous female alaskan tanner crab (Chionoecetes bairdi). Journal of Crustacean Biology v.3, p. 183 186, 1983.

STERNBERG, R. V. Systematic implications of color pattern polymorphism in Goniopsis pulchra (Decapoda: Brachyura: Grapsidae) from Ecuador. Proceedings of the Biology Society of Washington, v. 107, n. 4, p. $721-728,1994$.

Tropical Oceanography, Recife: v. 31, n.2, p. 127-133, 2003 
VALENTI, W. C.; MELLO, J. T. C.; LOBÃO, V. L. Fecundidade em Macrobrachium acanthurus (Wiegman, 1836) do rio Ribeira de Iguape (Crustacea, Decapoda, Palaemonidae). Revista Brasileira de Zoologia v. 6, n. 1, p. 9 - 15, 1989.

WICKHAM, D.E. Predation by nemertean Carcinonemertes errans on eggs og the dungeness crab Cancer magister. Marine Biology v. 55, p. 45 - 53, 1979.

Tabela I - Fecundidade por classe de tamanho de fêmeas ovígeras de Goniopsis cruentata capturadas no manguezal do rio Paripe - Pernambuco, no período de agosto/1999 a julho/2000.

\begin{tabular}{|c|c|c|c|}
\hline $\begin{array}{c}\text { Classe de } \\
\text { Tamanho } \\
(\mathrm{mm})\end{array}$ & $\mathbf{n}$ & $\begin{array}{c}\mathrm{n}^{0} \text { de ovos por } \\
\text { fêmeas (média) }\end{array}$ & $\begin{array}{l}\text { Desvio } \\
\text { Padrão }\end{array}$ \\
\hline $28-32$ & 4 & 51.960 & 7,7 \\
\hline $32-36$ & 14 & 75.390 & 21,7 \\
\hline $36-40$ & 12 & 92.370 & 5,8 \\
\hline $40-44$ & 12 & 91.740 & 9,5 \\
\hline $44-48$ & 3 & 144.380 & 42,1 \\
\hline
\end{tabular}

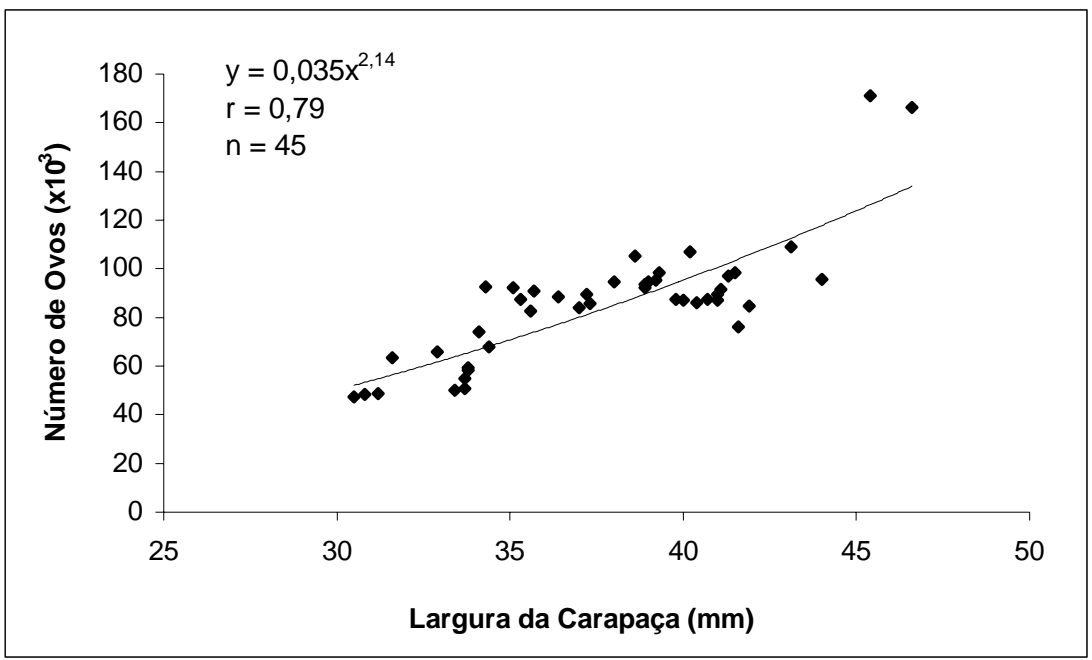

Figura 1 - Relação entre a largura da carapaça e o número de ovos em fêmeas ovígera de Goniopsis cruentata capturadas no manguezal do rio Paripe - Pernambuco, no período de agosto/1999 a julho/2000. 


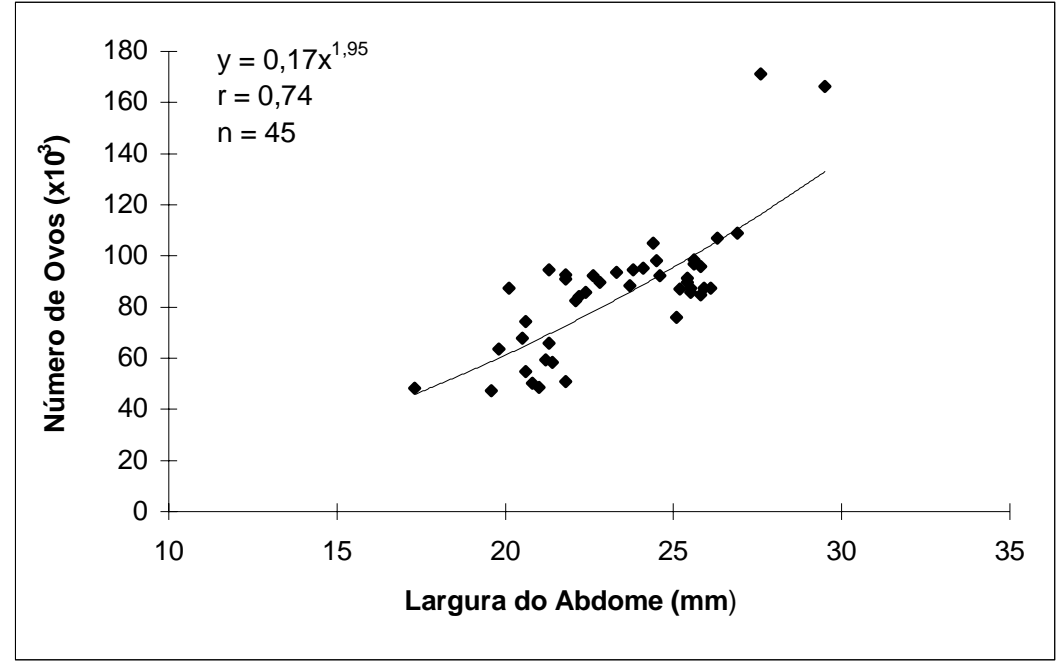

Figura 2 - Relação entre a largura do abdome e o número de ovos em fêmeas ovígera de Goniopsis cruentata capturadas no manguezal do rio Paripe - Pernambuco, no período de agosto/1999 a julho/2000. 
Tropical Oceanography, Recife: v. 31, n. 2, 2003 